\title{
Selective ablation of immature blood vessels in established human tumors follows vascular endothelial growth factor withdrawal
}

\author{
Laura E. Benjamin, ${ }^{1}$ Dragan Golijanin, ${ }^{2}$ Ahuva Itin, ${ }^{1}$ Dov Pode,${ }^{2}$ and Eli Keshet ${ }^{1}$ \\ ${ }^{1}$ Department of Molecular Biology, The Hebrew University/Hadassah Medical School, Jerusalem 91120, Israel \\ ${ }^{2}$ Department of Urology, Haddash University Hospital, Jerusalem 91120, Israel \\ Address correspondence to: Eli Keshet, Department of Molecular Biology, The Hebrew University/Hadassah Medical School, \\ Jerusalem 91120, Israel. Phone: (972) 2-6758496; Fax: (972) 2-6757195; E-mail: keshet@cc.huji.ac.il
}

Received for publication August 24, 1998, and accepted in revised form November 19, 1998.

Features that distinguish tumor vasculatures from normal blood vessels are sought to enable the destruction of preformed tumor vessels. We show that blood vessels in both a xenografted tumor and primary human tumors contain a sizable fraction of immature blood vessels that have not yet recruited periendothelial cells. These immature vessels are selectively obliterated as a consequence of vascular endothelial growth factor (VEGF) withdrawal. In a xenografted glioma, the selective vulnerability of immature vessels to VEGF loss was demonstrated by downregulating VEGF transgene expression using a tetracycline-regulated expression system. In human prostate cancer, the constitutive production of VEGF by the glandular epithelium was suppressed as a consequence of androgen-ablation therapy. VEGF loss led, in turn, to selective apoptosis of endothelial cells in vessels devoid of periendothelial cells. These results suggest that the unique dependence on VEGF of blood vessels lacking periendothelial cells can be exploited to reduce an existing tumor vasculature.

J. Clin. Invest. 103:159-165 (1999).

\section{Introduction}

The concept of treating tumors by inhibiting their ability to recruit new blood vessels is based on the premise that tumors are angiogenesis-dependent (1) and will remain dormant if neovascularization is prevented (2). The feasibility of antiangiogenic tumor therapy has been demonstrated by a number of animal studies, using either agents that antagonize a positive regulator of angiogenesis, primarily vascular endothelial graowth factor (VEGF) (3-7), or the administration of a negative regulator of angiogenesis, e.g., angiostatin and endostatin (8-10). Importantly, antiangiogenic therapy of experimental cancer does not induce acquired drug resistance (11). The general quiescence of the non-tumor endothelium (12) is in contrast to the state of ongoing angiogenesis and vascular remodeling taking place in tumors, and suggests that antiangiogenic therapy might not be detrimental to the nontumor vasculature.

Ideally, however, treating an established human tumor might require not only the inhibition of further angiogenesis but also loss of existing tumor vessels to reduce the existing tumor mass. To accomplish this, features that distinguish a pre-formed tumor vasculature from host vessels are sought, and one such feature might be the state of vessel maturation. We have shown recently that during development immature vessels differ from mature ones in their dependence on VEGF for survival. Thus, whereas VEGF is required to sustain newly formed vessels, this survival factor is dispensable for the mature vascular network (13). During development, the transient existence of a pericyte-free endothelial plexus allows for oxygen-regulated pruning (mediated by VEGF) as a mechanism for determining the final vascular density according to the needs of the surrounding tissue. Importantly, the acquisition of a pericyte coating marks the end of this plasticity window and stabilizes the vasculature (14).

Prior studies of embryogenesis have highlighted the essential role of periendothelial cells in maintaining vascular integrity, including the disruption of the genes encoding the endothelial cell-specific receptors Tie-1 and Tie-2 (15-17), the Tie-2 ligand angiopoetin-1 (Ang1) (18), the tissue factor (TF) system (19), and the platelet-derived growth factor (PDGFB)/PDGF- $\beta$ receptor system (20-22).

We reasoned that, because of ongoing angiogenesis and remodeling of tumor vessels, there is a fraction of tumor vessels that have not yet recruited periendothelial cells. Indeed, we show that well-established human tumors contain a significant fraction of vessels devoid of periendothelial cells. We then examine the thesis that, because of their dependence on vascular survival factors like VEGF, these vessels can be forced to regress. The feasibility of forcing a selective regression of immature vessels is first demonstrated using a genetic system designed for conditional VEGF expression in a xenografted glioma tumor. To examine the feasibility of this approach in a well-established, slow-growing human tumor, we analyzed prostate cancer specimens.

Growth of the prostate in rodents seems to be regulated by vascular endothelial cells, which themselves are responding to angiogenic/trophic factors elaborated by the prostate epithelium under testosterone stimulation (23). VEGF, in particular, is produced by the secretory epithelium of normal, hyperplastic, and tumorous prostate glands $(24,25)$. Expression of VEGF in androgen-dependent cell lines is downregulated upon androgen withdrawal, and in tumors composed of these cells, 

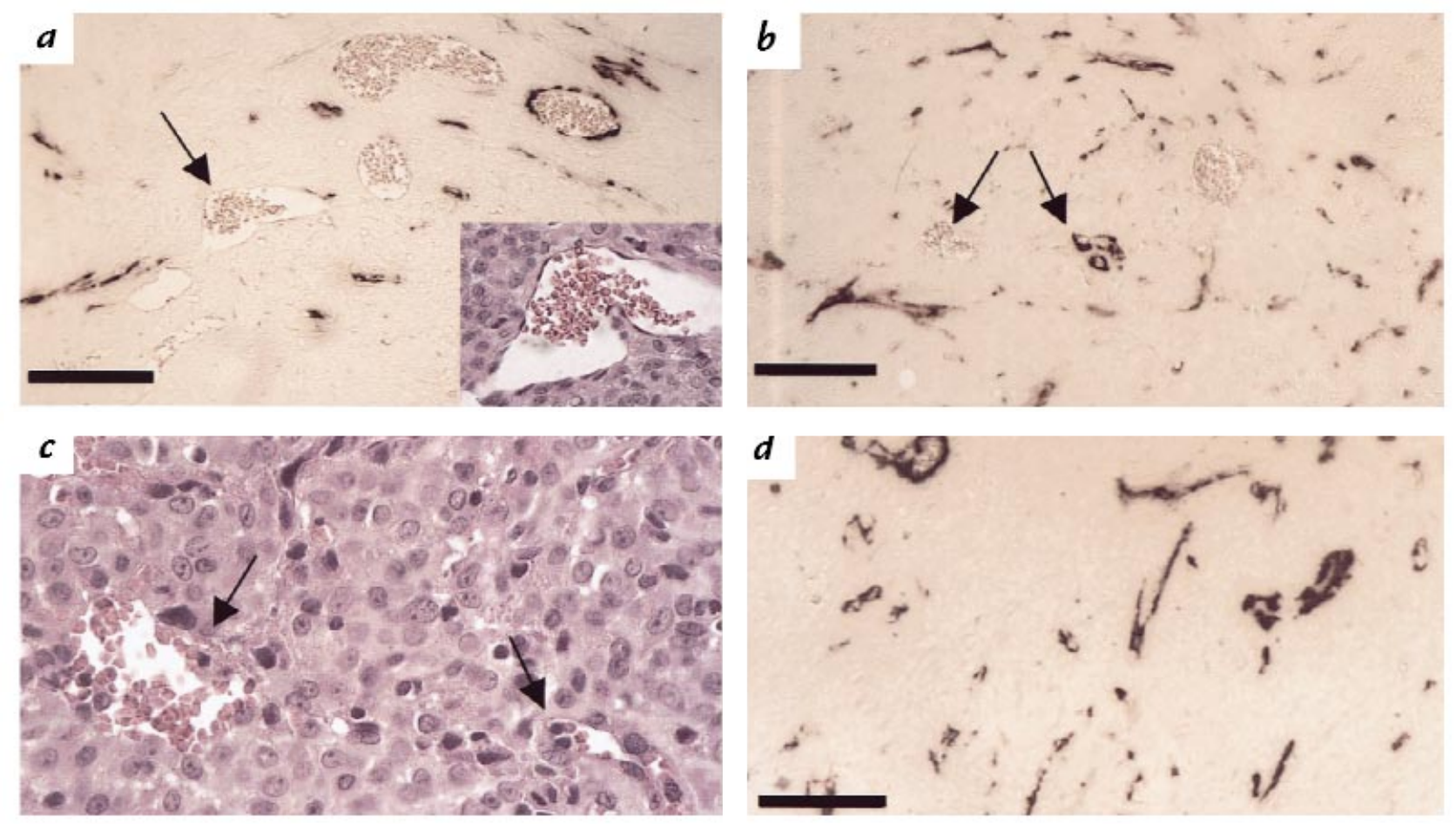

\section{Figure 1}

Regression of immature blood vessels in a xenografted glioma tumor. (a) A tumor grown under conditions of constitutive high-VEGF expression showing a mixture of $\alpha$-SMA-positive and $\alpha$ SMA-negative blood vessels. The $\alpha$-SMA-negative vessel (arrow) is shown at a higher magnification in the inset (counterstained with H\&E) to highlight the integrity of its endothelium. ( $\boldsymbol{b}$ and c) A tumor $72 \mathrm{~h}$ after VEGF withdrawal. Both covered (right arrows) and uncovered (left arrows) vessels are still observed. However, the uncovered vessel shows clear evidence of disintegration (better seen in $c$ depicting the same vessel in a serial section counterstained with $\mathrm{H} \& \mathrm{E}$ ). (d) A tumor 5 days after VEGF withdrawal. Note that most surviving vessels are $\alpha$-SMA-positive. (e) VMIs were determined, as described in Methods, in high-power fields of sections obtained either before or 5 days after VEGF withdrawal (scoring 270 or 87 vessels, respectively). Calculated VMIs were $0.30(\mathrm{SEM}=0.04)$ and $0.94(\mathrm{SEM}=0.05)$, respectively. $H \& E$, hematoxylin and eosin; SMA, smooth muscle actin; $V E G F$, vascular endothelial growth factor; $V M I$, vessel maturation indices.

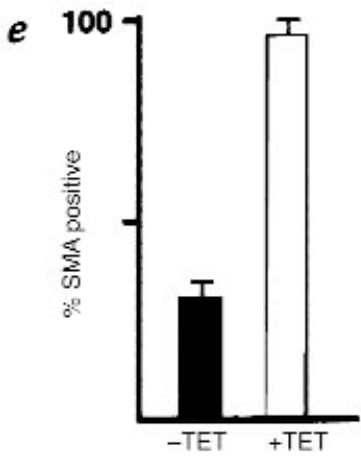

castration leads to vascular regression, which precedes tumor cell death (26). In prostate cancer patients, total androgen-ablation therapy is a common practice, intended to reduce the tumor volume and downstage operable tumors. Availability of clinical materials of untreated and androgen-ablated prostate tissue provided us with a unique opportunity to examine the consequences of VEGF loss in a natural, well-established human tumor. We show here that immature vessels are selectively eliminated by androgen ablation, thereby providing a new mechanistic insight into this mode of therapy.

\section{Methods}

Xenograft tumors with tetracycline-regulated VEGF expression. C6 glioma cells expressing a transfected VEGF gene under tetracycline control were used. Xenograft glioma cells were grown subcutaneously in nude mice until the tumor was $0.5-1.0 \mathrm{~cm}^{3}$. Tetracycline was administered to the drinking water of animals as described previously (27). Tumors were fixed in formalin and embedded in paraffin for analysis.

Immunohistochemistry. Paraffin-embedded tumor sections were stained for $\alpha$-smooth muscle actin ( $\alpha$-SMA) (Sigma Chemical Co.,
St. Louis, Missouri, USA) to detect vascular smooth muscle cells and pericytes. Blood vessels were detected by either Bandeiraea simplicifolia BS-1 Isolectin (LEC) (mouse tumors) or anti-factor VIII, von Willebrand factor (vWF) (human tumors). The vWF was used in a 1:1,000 dilution after trypsinization for $30 \mathrm{~min}$ at $37^{\circ} \mathrm{C}$. Secondary antibodies used were conjugated to either horseradish peroxidase or alkaline phosphatase, and conjugates were visualized with 3-aimino-9-ethyl carbasole (AEC) (Sigma Chemical Co.) or nitroblue tetrazolium/5-bromo-4-chloro-3-indolyl phosphate (Promega Corp., Madison, Wisconsin, USA), respectively. Control staining, excluding primary antibody, was carried out in each case. For fluorescent immunostaining, anti-mouse FITC (for $\alpha$-SMA) and anti-mouse tetrarhodamine isothiocyanate [TRITC] (for terminal deoxynucleotide transferase mediated dUTP nick end labeling [TUNEL]) antibodies were used. 4,6-diamidino-2-phenylindole (DAPI) staining was included in mounting medium. TUNEL analysis was carried out as described previously (27). The fraction of blood vessels found to be associated with $\alpha$-SMA-positive cells was defined as the vessel maturation index.

Hormone ablation. Human materials used in this study were all in the form of archival paraffin blocks. Cases selected for this study were according to their medical history. Patients were treated for hormone ablation before radical prostatecto- 
my by luteinizing hormone-releasing hormone agonist (Zoladex [Goserelin], Zeneca Pharmaceuticals, Wilmslow, United Kingdom) in combination with anti-androgen (Eulexin [Flutamide] Schering-Plough, Madison New Jersey, USA). In this course, serum androgen levels are effectively eliminated after 4 weeks, and treatment was continued for an additional 4-8 weeks before surgery.

Microscopy and imaging. Conventional fluorescent and light microscopy was performed on a Zeiss Axioskop (Carl Zeiss Jena Gmbh, Jena Germany). Slide film was scanned into Photoshop 3.0 (Adobe Systems Inc., Mountain View, California, USA) Confocal microscopy was performed on a Zeiss Axiovert 135M (Carl Zeiss Jena Gmbh) outfitted with the software program LSM410. Double immunofluorescence with FITC and TRITC were visualized using filters BP510-525 and LP570.

In situ bybridization. Paraffin-embedded specimens from archival pathological materials were hybridized in situ with a VEGF-specific probe as described previously (28). Autoradiographic exposure was for 6 days.

\section{Results}

VEGF deprivation leads to selective obliteration of immature vessels in a xenografted glioma tumor. To enable control of the level of VEGF elaborated by tumor cells, a conditional VEGF expression system was created in which expression of VEGF by a C6 glioma is repressed when tetracycline is added to the drinking water of tumorbearing nude mice. With the aid of this system, we have shown previously that overexpression of VEGF results in hypervascularization and that VEGF contributed by the endogenous gene was insufficient to sustain all vessels generated under conditions of VEGF overexpression. The latter is evident by the finding that shutting off VEGF production from the transgene resulted in detachment of a fraction of endothelial cells from the walls of some preformed vessels and their subsequent death by apoptosis. Vascular collapse led, in turn, to massive hemorrhages and extensive tumor necrosis (27). This system was used here to determine whether vulnerability to VEGF deprivation is associated with the state of vessel maturation.

Tumors were first allowed to grow for several weeks under conditions of maximal VEGF expression. After resection we determined the vessel maturation index, defined as the fraction of vessels that are associated with $\alpha$-SMA-positive periendothelial cells. This measure was determined by scoring blood vessels larger than capillaries (i.e., vessels containing a lumen large enough for several erythrocytes), because capillaries are sparsely coated by pericytes and, therefore, may falsely appear as uncovered in thin sections. $\alpha$-SMA-positive periendothelial cells could not be detected in the majority of these vessels, indicating that a large fraction of tumor blood vessels were immature (Fig. 1a). VEGF production from the transgene was then switched off in the remainder of the tumor-bearing animals. We have shown previously that this protocol leads to almost complete abrogation of transgene expression (27). Intermediate stages in vessel regression could be detected at 72 hours after switching off VEGF production and were evident by a loss in the continuity of the endothelial cell lining and by erythrocyte escape. Strikingly, evidence of vessel injury was only detected in vessels devoid of a periendothelial cell coating, including cases where $\alpha$-SMA-negative vessels and $\alpha$-SMA-positive vessels occupied the same tumor niche (Fig. 1, $b$ and $c$ ). The outcome of this differential vulnerability was that by five days after VEGF withdrawal, the majority of the surviving vessels were found to have acquired an smooth muscle cell/pericyte coating (Fig. 1d; see also $e$ for quantification of increase in the vessel maturation index). This result indicated that only immature tumor vessels depend on soluble VEGF for survival.

Established human tumors contain a significant fraction of ves-
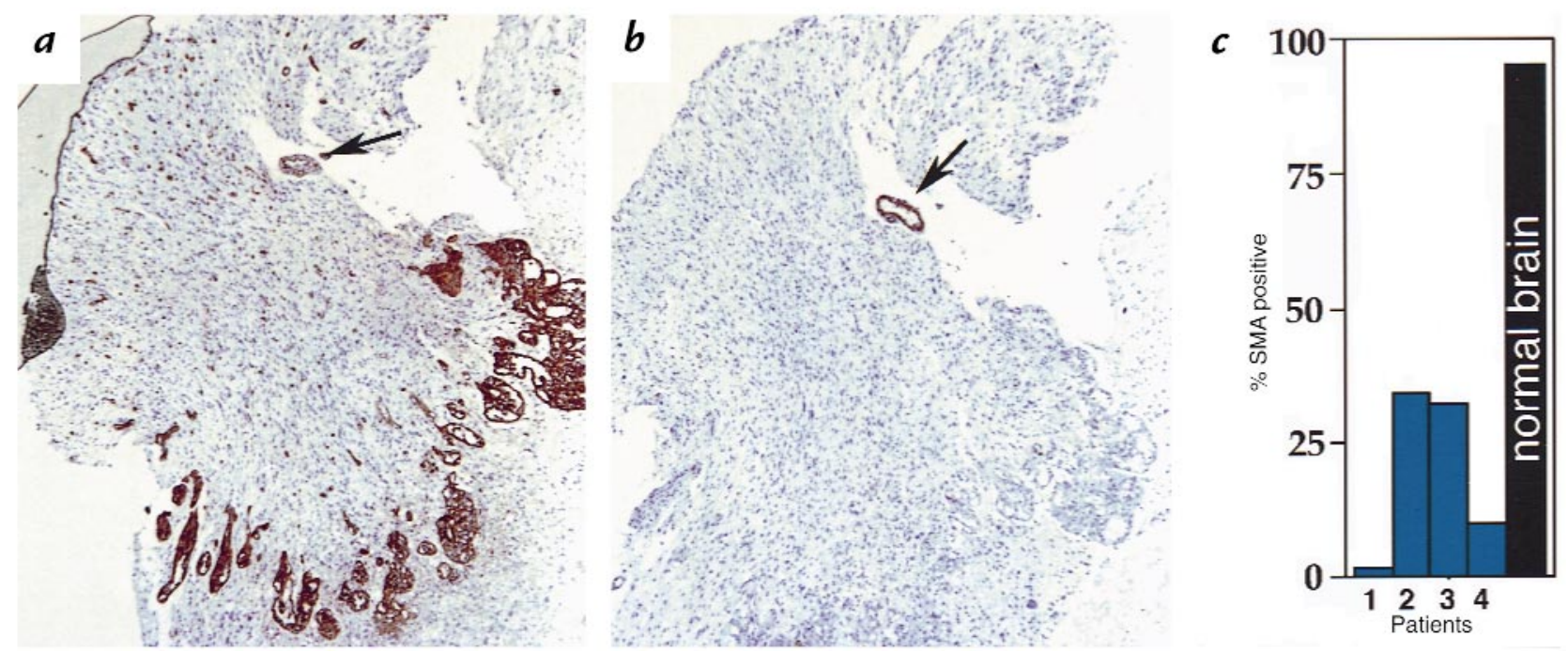

Figure 2

Most blood vessels in glioblastoma multiforme are immature. ( $\boldsymbol{a}$ and $\boldsymbol{b}$ ) Serial sections of a glioblastoma multiforme tumor stained with anti-vWF (a) and with anti- $\alpha$-SMA (b) showing only few $\alpha$-SMA-positive vessels (arrows). (c) Four glioblastoma specimens were serially stained for $\alpha$-vWF and $\alpha$-SMA. Vessels larger than capillaries were scored (between 58 and 212 for each specimen), and the percentage of $\alpha$-SMA-positive vessels is presented (averaging 19\%; SEM $=8.3 \%$ ). For comparison, vessels of a normal adult brain (rat) were also evaluated for the percentage of $\alpha$-SMA-positive vessels (average of six high-power fields was 95\%; SEM $=3 \%$ ). $v W F$, von Willebrand factor. 


\section{Figure 3}

Downregulation of prostatic VEGF mRNA expression by androgen-ablation therapy. (a) In situ hybridization of a neoplastic prostate specimen with a VEGF-specific probe shown at high magnification. Note abundant expression of VEGF in the abnormal glandular epithelium. (b-e) Lowpower magnifications to show global changes in VEGF expression via in situ hybridization of grade-matched specimens either untreated $(b$, brightfield; $d$, dark-field) or subjected to androgen-ablation treatment before prostatectomy ( $c$, bright-field; $e$, dark-field). Sections were cohybridized on the same slide. Note a marked reduction in VEGF expression as a result of androgen-ablation therapy. sels devoid of periendothelial cells. Before considering the targeting of immature tumor vessels it was essential to show that, like in the xenografted glioma models, natural human tumors contain a significant fraction of immature vessels. To determine the fraction of immature vessels present in a well-established glioblastoma, pathological specimens of glioblastoma multiforme were analyzed for the fraction of vessels that are $\alpha$-SMA-positive. A representative field is shown in Fig. 2, depicting a high density of glomerulus-like vessels (a hallmark of glioblastoma multiforme) and smaller blood vessels nearby. Most of these blood vessels lack $\alpha$-SMA-positive periendothelial cells. In the glioblastoma specimens examined, on average, less than one-quarter of tumor vessels larger than
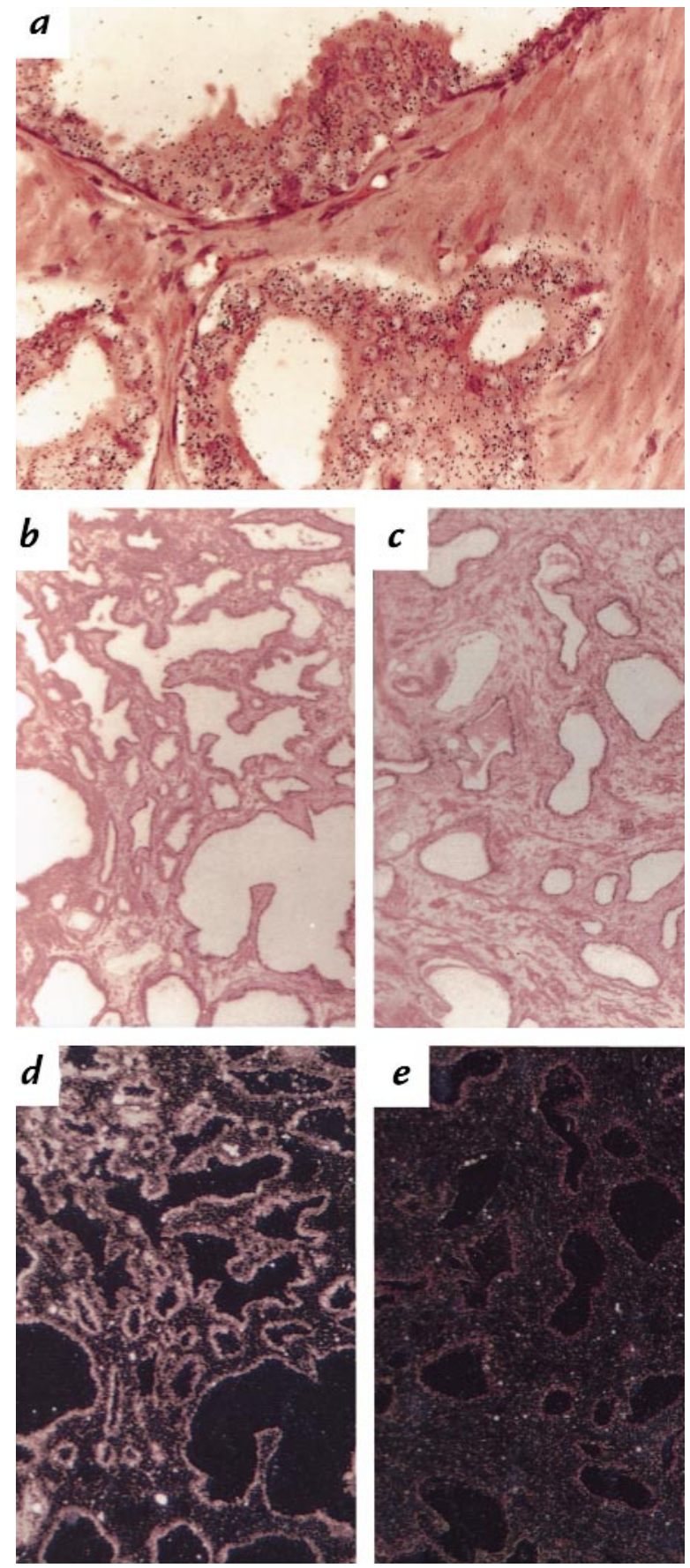

capillaries were associated with $\alpha$-SMA-positive cells . The presence of a large fraction of immature vessels in glioblastoma multiforme was in sharp contrast to the normal brain where the majority of vessels were covered by $\alpha$-SMA-positive cells (Fig. $2 c$ ). Glioblastoma multiforme is a fast-growing and highly angiogenic tumor. Therefore, it could be argued that the high proportion of immature vessels in this tumor reflects an exceptionally high rate of tumor growth and neovascularization and that slow-growing tumors may contain only an insignificant fraction of immature vessels.

To represent a slow-growing tumor, we analyzed prostate carcinoma specimens obtained from radical prostatectomies. Fields selected for vessel counting were 

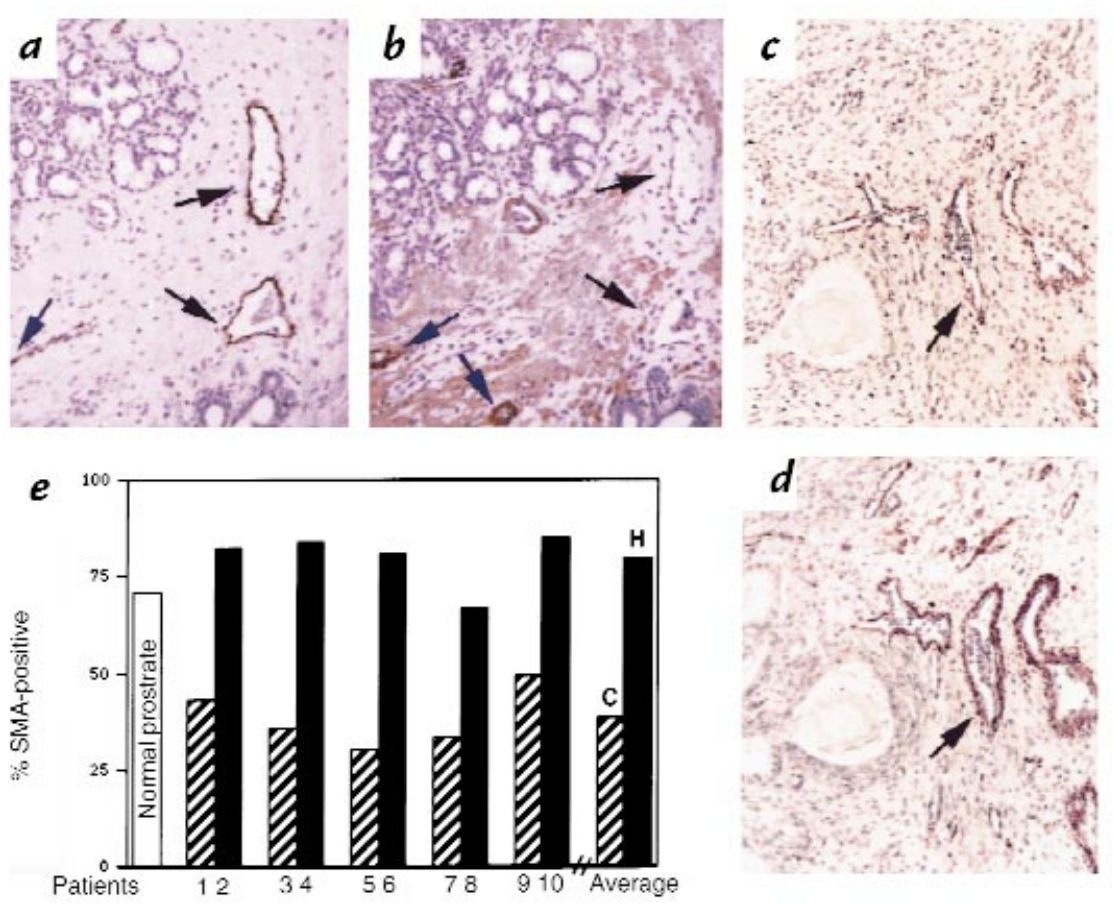

Figure 4

Androgen-ablation therapy in prostate cancer leads to selective obliteration of immature vessels. Adjacent sections of surgically removed prostate tissues were immunostained for VWF ( $\boldsymbol{a}$ and $\boldsymbol{c}$ ) or for $\boldsymbol{\alpha}$-SMA ( $\boldsymbol{b}$ and $\boldsymbol{d}$ ) to examine individual vessels for coverage with periendothelial cells. One example is shown for an untreated tumor $(a$ and $b$ ) (black arrows pointing at uncovered vessels and a blue arrow pointing at a covered vessel) and one for a tumor subjected to androgen-ablation therapy $(c$ and $d$ ) (black arrows pointing at a covered vessel). Data from 10 different patients are shown in the histogram $(\boldsymbol{e})$ : five from control untreated tumors (hatched bars) and five from treated tumors (solid bars). To randomize for experimental variability during processing and immunohistochemical detection, pairs of tumors, each containing one control tumor and an androgen-ablated tumor of the same Gleason grade and a matching patient age, were embedded in a single block and coanalyzed on the same slide. The total number of lumenized vessels scored depended on the amount of tumor represented in the section and was as follows: tumor 1 (140), 2 (63), 3 (25), 4 (19), 5 (98), 6 (122), 7 (158), 8 (143), 9 (170), 10 (397). Mouse prostate was used as a control for vascular maturation in normal prostate. On average, untreated tumors contained 38\% $\alpha$-SMA-positive vessels (SEM $=3.5 \%$ ); androgen-ablated tumors had 79\% $\alpha$-SMA-positive vessels $(S E M=3.3 \%)$.

centered around areas showing clear evidence of a carcinoma (as indicated by a specialized pathologist) and also contained normal and hyperplastic glands. On average, only $40 \%$ of vessels larger than capillaries were found to contain $\alpha$-SMA-positive cells. Thus, even in regions containing much normal tissue, almost half of the blood vessels were immature. Interestingly, the healthy prostate gland also contained a significant fraction of immature vessels (see Fig. 4e).

Androgen-ablation therapy in prostate cancer leads to VEGF loss and selective obliteration of immature vessels. Recent studies in animal models showing that androgen deprivation may lead to vascular regression, in conjunction with findings that VEGF is androgen-regulated, prompted us to examine whether androgen-ablation therapy in human prostate carcinoma might induce the selective obliteration of immature vessels that depend on VEGF for survival.

To show that VEGF in the human prostate is indeed downregulated by androgen-ablation therapy, in situ hybridization analysis with a VEGF-specific probe was carried out. In untreated specimens VEGF was found to be abundantly expressed by the secretory epithelium of normal, hyperplastic, and cancerous glands. An example is shown in Fig. 3a; it depicts expression of VEGF by the secretory epithelium in normal glands and prostatic intraepithelial neoplasia (PIN). Androgen regulation of VEGF was demonstrated by comparing specimens of rad- ical prostatectomy from untreated patients with those from patients subjected to androgen-ablation therapy for several weeks before surgery. In eight out of eight gradeand age-matched pairs cohybridized on the same slide, a dramatic reduction of VEGF expression was observed in the androgen-ablated specimens (for a representative example, compare Fig. 3, $c$ and $e$ with $b$ and $d$ ). Hybridization of adjacent sections with another gene known to be downregulated in the prostate after androgen derivation, neutral endopeptidase 24.11 (29), showed that both genes are coexpressed in the same cells and are coordinately downregulated in the absence of androgen (data not shown). While a retrospective analysis of surgically removed human tumors is short of proving a causal relationship between androgen ablation and VEGF downregulation, a similarity to the situation in an animal model (26) is consistent with this proposition.

To determine whether VEGF loss has led to selective elimination of immature prostatic vessels, the fraction of vessels coated by $\alpha$-SMA-positive cells was determined in untreated tumors and in tumors subjected to androgen-ablation therapy for several weeks before surgical removal. Shown in Fig. 4 are examples of the two types of vessels scored: vWF-positive/ $\alpha$-SMA-negative vessels (highlighted by black arrows in Fig. 4, $a$ and $b$ ), and vessels of a comparable size that are vWF-positive $/ \alpha$ SMA-positive (highlighted by black arrows in Fig. 4, $c$ 


\section{Figure 5}

Endothelial cell apoptosis after androgen ablation. TUNEL analysis was used to detect apoptotic cells in the untreated prostate and 4 weeks after hormone ablation. (a) An untreated prostate showing apoptotic nuclei (red) in glands but not in blood vessels (arrows). (b) An androgen-ablated specimen highlighting two blood vessels with several TUNEL-positive endothelial cells. Note the presence of TUNEL-positive (black arrow) and TUNEL negative (red arrow) in the same blood vessel. $a$ and $b$ were processed together on the same microscope slide to control for histochemical variability. (c) TUNEL (green fluorescence) and $\alpha$-SMA staining (red fluorescence) showing that an uncovered blood vessel (arrowhead) contains many apoptotic endothelial cells, whereas an adjacent covered blood vessel (arrow) does not. Red autofluorescence of erythrocytes aids in identifying the lumen of these vessels. Eighty-five percent of vessels in which one or more TUNEL-positive endothelial cells were detected were $\alpha$-SMA-negative. The mean number of TUNEL-positive endothelial cells per vessel was 3.1-fold greater in androgen-ablated tumors. TUNEL, terminal deoxynucleotide transferase-mediated dUTP nick end-labeling.

and $d$ ). Quantification of each type of vessel in several high-power fields of each tumor specimen allowed the assignment of a vessel maturation index to each tumor. It was anticipated that a preferential vulnerability of immature vessels, and hence their selective obliteration, would result in an increase in the vessel maturation index. Indeed, untreated tumors contained only $38 \%$ coated vessels, whereas tumors resected 8-12 weeks after the onset of androgen-ablation therapy contained $79 \%$ coated vessels, suggesting that mature vessels are more refractory to this treatment (Fig. 4e).

In conjunction with an in situ apoptosis (TUNEL) analysis, these pathological specimens provided an opportunity to observe intermediate stages in vessel regression. In untreated tumors, TUNEL-positive endothelial cells were only rarely detected, and apoptotic cells were more often detected in the glandular epithelium (presumably reflecting a normal turnover of these cells) (Fig. 5a). In contrast, endothelial cell apoptosis was frequently detected after androgen ablation (Fig. $5 b$ ). Most informative were sections in which $\alpha$-SMA-positive vessels were seen alongside $\alpha$-SMA-negative vessels. Strikingly, in these cases endothelial cells undergoing apoptosis were predominantly found in $\alpha$-SMA-negative vessels (Fig. 5c). These results provided conclusive evidence that a consequence of VEGF loss triggered by androgen deprivation is the selective obliteration of immature prostatic blood vessels.

\section{Discussion}

It is established that blocking angiogenesis can prevent tumor growth in animals. Additionally, the antiangiogenic agents angiostatin and endostatin have been shown to not only block tumor growth but cause tumor regression in xenograft cancer models through an unknown mechanism (11). We show here that the dependence of immature vessels on VEGF for survival can be exploited to reduce an existing tumor vasculature and thus reduce the dependent tissue mass. Using the recruitment of periendothelial cells as a hallmark of vessel maturation, we have shown in this study that wellestablished human tumors contain a sizable fraction of immature blood vessels. In a glioma model of conditional VEGF expression, we have seen that while only a fraction of blood vessels are sensitive to VEGF with-

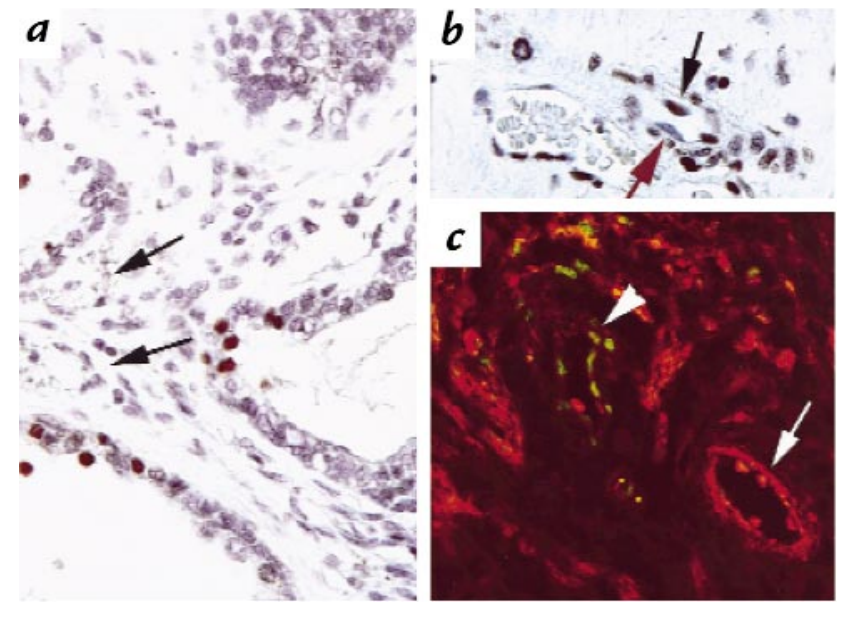

drawal, massive hemorrhage and necrosis results (27). The fact that these vessels are part of a closed, functional network may account for the great extent of hemorrhaging and tumor cell death.

Maintaining the option of vascular pruning for a certain period after the formation of the initial plexus normally serves the physiological role of adjusting vascular density to match oxygen supply. Accordingly, hyperoxia is the physiologic trigger for a developmental vessel regression, and whenever it causes downregulation of VEGF beyond the level required to sustain immature vessels, these vessels regress. An important element in this regulation is that the acquisition of pericytes stabilizes the vasculature, preventing deleterious vessel regression due to transient fluctuations in tissue oxygen. This study shows that suppression of VEGF production by means other than hyperoxia, namely by hormone deprivation, also leads to regression of immature vessels.

VEGF is abundantly expressed by the secretory epithelium of the human prostate and its production requires a continuous stimulation by androgen. The physiological role(s) of VEGF in the prostate is poorly understood and target cells may also include cells other than endothelial cells. However, with respect to the vasculature, it is clear that VEGF is required for maintenance of vascular homeostasis in the prostate, which by nature has a relatively high endothelial cell turnover (1\%-2\%) (30) and maintains a high fraction of immature vessels (Fig. 4e). The latter finding is likely responsible for the fact the prostate vasculature is so vulnerable to androgen-ablation therapy and can partially explain the reduction in tumor and normal glandular mass seen in the prostate after castration in animals (23). Anti-VEGF antibodies have been demonstrated to block tumor growth of prostate xenografts (7), and an antiangiogenic treatment with Linomide can be enhanced by simultaneous castration and downregulation of VEGF (31). It is not surprising that impairing the integrity of the tumor vasculature should have a strong impact on tumor cell viability, considering the paracrine effects mediated by a variety of growth and survival factors between the endothelium and tumor cell compartments (32).

These results also suggest that the vessel maturation index of a given tumor might predict the efficiency of an 
anti-VEGF treatment in reducing the tumor mass. Vessel diameter, on the other hand, does not seem to correlate with vulnerability/refractoriness to VEGF withdrawal, because vessels surviving VEGF shutoff in the C6 glioma system are not significantly different sizewise from vessels present before VEGF withdrawal. The fact that there is a big difference in the vessel maturation index of vessels in the normal brain and vessels in glioblastoma (Fig. 2c) suggests that this human tumor may respond well to anti-VEGF action.

We have shown previously that regression of VEGFdependent tumor vessels occurs via endothelial cell detachment (27). It seems likely that VEGF is required in a positive way to maintain cell adhesion to a provisional extracellular matrix until periendothelial cells facilitate a more permanent mode of adhesion. Therefore, it is possible that VEGF loss also effects the expression or activity of molecules mediating the adhesion of endothelial cells to the provisional matrix. A possible regulatory link might exist with the $\alpha \mathrm{V} / \beta 3$ and $\alpha \mathrm{V} / \beta 5$ integrins shown to be required for the survival of newly formed vessels $(33,34)$.

\section{Acknowledgments}

We thank pathologists D. Sofer and G. Pizov for help in evaluating glioblastoma and prostate carcinoma, and M. Kogan for excellent technical assistance. L.E. Benjamin is supported by the Israel Cancer Research Fund.

1. Folkman, J. 1997. Cancer: principles and practice of oncology. LippincottRaven Publishers. Philadelphia, PA. 3075-3085.

2. Holmgren, L., O'Reilly, M.S., and Folkman, J. 1995. Dormancy of micrometastases: balanced proliferation and apoptosis in the presence of angiogenesis suppression. Nat. Med. 1:149-153.

3. Millauer, B., et al. 1996. Dominant-negative inhibition of Flk-1 suppresses the growth of many tumor types in vivo. Cancer Res. 56:1615-1620.

4. Warren, R.S., Yuan, H., Matli, M.R., Gillett, N.A., and Ferrara, N. 1995. Regulation by vascular endothelial growth factor of human colon cancer tumorigenesis in a mouse model of experimental liver metastasis. J. Clin. Invest. 95:1789-1797.

5. Borgstrom, P., Hillan, K.J., Sriramarao, P., and Ferrara, N. 1996. Complete inhibition of angiogenesis and growth of microtumors by anti-vascular endothelial growth factor neutralizing antibody: novel concepts of angiostatic therapy from intravital videomicroscopy. Cancer Res. 56:4032-4039.

6. Yuan, F., et al. 1996. Time-dependent vascular regression and permeability changes in established human tumor xenografts induced by an anti-vascular endothelial growth factor/vascular permeability factor antibody. Proc. Natl. Acad. Sci. USA. 93:14765-14770.

7. Borgstrom, P., Bourdon, M.A., Hillan, K.J., Sriramarao, P., and Ferrara, N. 1998. Neutralizing anti-vascular endothelial growth factor antibody completely inhibits angiogenesis and growth of human prostate carcinoma micro tumors in vivo. Prostate. 35:1-10

8. O'Reilly, M.S., et al. 1997. Endostatin: an endogenous inhibitor of angiogenesis and tumor growth. Cell. 88:277-285.

9. O'Reilly, M.S., et al. 1994. Angiostatin: a novel angiogenesis inhibitor that mediates the suppression of metastases by a Lewis lung carcinoma [comment]. Cell. 79:315-328.

10. O'Reilly, M.S., Holmgren, L., Chen, C., and Folkman, J. 1996. Angiostatin induces and sustains dormancy of human primary tumors in mice. Nat. Med. 2:689-692.
11. Boehm, T., Folkman, J., Browder, T., and O’Reilly, M.S. 1997. Antiangiogenic therapy of experimental cancer does not induce acquired drug resistance. Nature. 390:404-407.

12. Denekamp, J. 1993. Angiogenesis, neovascular proliferation and vascular pathophysiology as targets for cancer therapy [review]. Br. J. Radiol. 66:181-196.

13. Alon, T., et al. 1995. Vascular endothelial growth factor acts as a survival factor for newly formed retinal vessels and has implications for retinopathy of prematurity. Nat. Med. 1:1024-1028.

14. Benjamin, L., Hemo, I., and Keshet, E. 1998. A plasticity window for blood vessel remodelling is defined by pericyte coverage of the preformed endothelial network and is regulated by PDGF-B and VEGF. Development. 125:1591-1598.

15. Dumont, D.J., et al. 1994. Dominant-negative and targeted null mutations in the endothelial receptor tyrosine kinase, tek, reveal a critical role in vasculogenesis of the embryo. Genes Dev. 8:1897-1909.

16. Puri, M.C., Rossant, J., Alitalo, K., Bernstein, A., and Partanen, J. 1995. The receptor tyrosine kinase TIE is required for integrity and survival of vascular endothelial cells. EMBO J. 14:5884-5891.

17. Sato, T.N., et al. 1995. Distinct roles of the receptor tyrosine kinases Tie1 and Tie-2 in blood vessel formation. Nature. 376:70-74.

18. Suri, C., et al. 1996. Requisite role of angiopoietin-1, a ligand for the TIE2 receptor, during embryonic angiogenesis [comment]. Cell. 87:1171-1180.

19. Carmeliet, P., et al. 1996. Role of tissue factor in embryonic blood vessel development. Nature. 383:73-75.

20. Leveen, P., et al. Mice deficient for PDGF B show renal, cardiovascular, and hematological abnormalities. Genes Dev. 8:1875-1887.

21. Lindahl, P., Johansson, B.R., Leveen, P., and Betsholtz, C. 1997. Pericyte loss and microaneurysm formation in PDGF-B-deficient mice. Science. 277:242-245.

22. Soriano, P. 1994. Abnormal kidney development and hematological disorders in PDGF beta-receptor mutant mice. Genes Dev. 8:1888-1896.

23. Franck-Lissbrant, I., Haggstrom, S., Damber, J.-E., and Bergh, A. 1988. Testosterone stimulates angiogenesis and vascular regrowth in the ventral prostate in castrated rats. Endocrinology. 139:451-456.

24. Jackson, M.W., Bentel, J.M., and Tilley, W.D. 1997. Vascular endothelial growth factor (VEGF) expression in prostate cancer and benign prostatic hyperplasia. J. Urol. 157:2323-2328.

25. Ferrer, F.A., et al. 1998. Angiogenesis and prostate cancer: in vivo and in vitro expression of angiogenesis factors by prostate cancer cells. Urology. 51:161-167.

26. Jain, R.K., et al. 1998. Endothelial cell death, angiogenesis and microvascular function following castration in an androgen-dependent tumor: role of VEGF. Proc. Natl. Acad. Sci. USA. 181:10820-10825.

27. Benjamin, L.E., and Keshet, E. 1997. Conditional switching of vascular endothelial growth factor (VEGF) expression in tumors: Induction of endothelial cell shedding and regression of hemangioblastoma-like vessels by VEGF withdrawal. Proc. Natl. Acad. Sci. USA. 94:8761-8766.

28. Pe'er, J., et al. 1995. Hypoxia-induced expression of vascular endothelial growth factor by retinal cells is a common factor in neovascularizing ocular diseases. Lab. Invest. 72:638-645.

29. Papandreou, C.N., et al. 1998. Neutral endopeptidase 24.11 loss in metastatic human prostate cancer contributes to androgen-independent progression. Nat. Med. 4:50-57.

30. English, H., Drago, J., and Santen, R. 1985. Cellular response to androgen depletion and repletion in the rat ventral prostate: autoradiographic and morphometrical analysis. Prostate. 7:41-51.

31. Joseph, I.B., and Isaacs, J.T. 1997. Potentiation of the antiangiogenic ability of linomide by androgen ablation involves down-regulation of vascular endothelial growth factor in human androgen-responsive prostatic cancers. Cancer Res. 57:1054-1057.

32. Rak, J., Filmus, J., and Kerbel, R. 1996. Reciprocal paracrine interactions between tumour cells and endothelial cells: the "angiogenic progression" hypothesis. Eur. J. Cancer. 32A:2438-2450.

33. Brooks, P.C., Clark, R.A., and Cheresh, D.A. 1994. Requirement of vascular integrin alpha $v$ beta 3 for angiogenesis. Science. 264:569-571.

34. Friedlander, M., et al. 1995. Definition of two angiogenic pathways by distinct alpha $v$ integrins. Science. 270:1500-1502. 